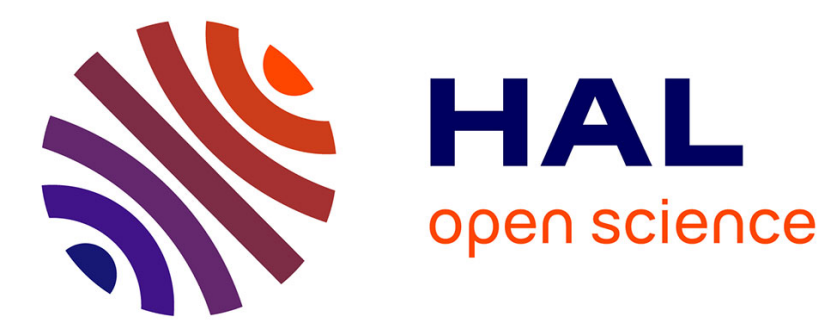

\title{
Component Importance Measures for Components With Multiple Dependent Competing Degradation Processes and Subject to Maintenance
}

Yan-Hui Lin, Yan-Fu Li, Enrico Zio

\section{- To cite this version:}

Yan-Hui Lin, Yan-Fu Li, Enrico Zio. Component Importance Measures for Components With Multiple Dependent Competing Degradation Processes and Subject to Maintenance. IEEE Transactions on Reliability, 2016, 65 (2), pp.547-557. 10.1109/TR.2015.2500684 . hal-01342098

\section{HAL Id: hal-01342098 \\ https://hal.science/hal-01342098}

Submitted on 18 Jul 2016

HAL is a multi-disciplinary open access archive for the deposit and dissemination of scientific research documents, whether they are published or not. The documents may come from teaching and research institutions in France or abroad, or from public or private research centers.
L'archive ouverte pluridisciplinaire HAL, est destinée au dépôt et à la diffusion de documents scientifiques de niveau recherche, publiés ou non, émanant des établissements d'enseignement et de recherche français ou étrangers, des laboratoires publics ou privés. 


\title{
Component Importance Measures for Components with Multiple Dependent Competing Degradation Processes and Subject to Maintenance
}

\author{
Yan-Hui Lin, Yan-Fu Li,senior member IEEE, Enrico Zio,senior member IEEE ${ }^{\mathbf{1}}$
}

Index Terms - Degradation dependency, importance measures, multiple dependent competing degradation processes, piecewise-deterministic Markov process (PDMP), finitevolume approach, residual heat removal system, nuclear power plant.

\begin{abstract}
Component importance measures (IMs) are widely used to rank the importance of different component within a system and guide allocation of resources. The criticality of a component may vary over time, under the influence of multiple dependent competing degradation processes and maintenance tasks. Neglecting this may lead to inaccurate estimation of the component IMs and inefficient related decisions (e.g. maintenance, replacement, etc.). The work presented in this paper addresses the issue by extending the mean absolute deviation IM by taking into account: (1) the dependency of multiple degradation processes within one component and among different components; (2) discrete and continuous degradation processes; (3) two types of maintenance tasks: condition-based preventive maintenance via periodic inspections and corrective maintenance.Piecewisedeterministic Markov processes are employed to describe the stochastic process of degradation of the component under these factors. A method for the quantification of the component IM is developed based on the finite-volume approach. A case study on one section of the residual heat removal system of a nuclear power plant is considered as an example for numerical quantification.
\end{abstract}

Y.H.Lin and Y.F.LiarewiththeChair System Science and the Energy Challenge, FondationElectricité de France (EDF), CentraleSupélec, Université Paris-Saclay, Grande Voie des Vignes, 92290 Chatenay-Malabry, France (e-mail:

yanhui.lin@centralesupelec.fr;yanfu.li@ centralesupelec.fr)

E. Zio is with theChair System Science and the Energy Challenge, FondationElectricité de France (EDF), CentraleSupélec, Université Paris-Saclay, Grande Voie des Vignes, 92290 Chatenay-Malabry, France, and also with the Politecnico di Milano, 20133 Milano, Italy (email: enrico.zio@centralesupelec.fr; enrico.zio@polimi.it) 


\section{Acronyms}

IMs Importance measures

PBMs Physics-based models

MSMs Multi-state models

GSA Global sensitivity analysis

BIM Birnbaum IM

MAD Mean absolute deviation

MSSs Multi-state systems

PM Preventive maintenance

$\mathrm{CM} \quad$ Corrective maintenance

FV Finite-volume

RHRS Residual heat removal system

\section{Notations}

Number of components in the system

$\boldsymbol{L}$

Group of degradation processes modeled by PBMs

$\boldsymbol{K}$

Group of degradation processes modeled by MSMs

$\boldsymbol{D}_{O_{q}}$

Degradation state of component $O_{q}$

$\overrightarrow{X_{L_{m}}}(t)$

Time-dependent continuous variables of degradation process $L_{m}$

$\overrightarrow{X_{L_{m}}^{\boldsymbol{D}}}(t)$

Non-decreasing degradation variables vector

$\overrightarrow{X_{L_{m}}^{P}}(t)$

Physical variables vector

$\mathcal{F}_{L_{m}}$

Set of failure states of degradation process $L_{m}$

$Y_{K_{n}}(t)$

State variable of degradation process $K_{n}$

$\boldsymbol{S}_{K_{n}}$

Finite state set of degradation process $K_{n}$ 


\begin{tabular}{|c|c|}
\hline $\mathcal{F}_{K_{n}}$ & Set of failure states of degradation process $K_{n}$ \\
\hline $\boldsymbol{H}_{i}$ & Predefined state set of PM for degradation process $i$ \\
\hline$T_{i}$ & Fixed period of PM for degradation process $i$ \\
\hline$\vec{Z}(t)$ & Degradation state of the system \\
\hline$N_{m}$ & Number of maintenance tasks experienced by the system \\
\hline$T_{\text {miss }}$ & System mission time \\
\hline$T_{m}^{k}$ & Execution time of the $k$-th maintenance task \\
\hline $\overrightarrow{Z_{k}}(t)$ & Degradation state of the system defined on $\left[T_{m}^{k-1}, T_{m}^{k}\right]$ \\
\hline $\boldsymbol{\theta}_{K}$ & Environmental and operational factors in $\boldsymbol{K}$ \\
\hline$\lambda_{\vec{i}}\left(\vec{\jmath} \mid \vec{X}(t), \boldsymbol{\theta}_{K}\right)$ & Transition rate from state $\vec{\imath}$ to $\vec{\jmath}$ \\
\hline $\boldsymbol{\theta}_{L}$ & Environmental and operational factors in $\boldsymbol{L}$ \\
\hline $\overrightarrow{f_{L}}\left(\overrightarrow{Z_{k}}(t), t \mid \boldsymbol{\theta}_{\boldsymbol{L}}\right)$ & Deterministic physics equations in $\boldsymbol{L}$ \\
\hline $\overrightarrow{Z^{\prime}}(t)$ & Stochastic process recording the failure of the system \\
\hline $\mathcal{F}$ & System failure state set \\
\hline$C I_{O_{q}}(t)$ & Component IM of component $O_{q}$ at time $t$ \\
\hline$f_{\overrightarrow{\boldsymbol{D}_{o_{q}}}(t)}\left(d \overrightarrow{\boldsymbol{x}_{\mathbf{L}_{p}}}, \overrightarrow{y_{\boldsymbol{K}_{q}}}\right)$ & Probability distribution of $\overrightarrow{\boldsymbol{D}_{O_{q}}}(t)$ \\
\hline \multicolumn{2}{|c|}{$p_{t}^{\overrightarrow{Z_{k}}}(d \vec{x}, \vec{\imath} \mid \boldsymbol{\theta})$ Probability distribution of processes $\overrightarrow{Z_{k}}(t)$} \\
\hline $\begin{array}{l}P_{n}^{\overrightarrow{Z_{k}}}(A, \vec{\imath} \mid \boldsymbol{\theta}) \\
\left\{\left(A^{k-1}, \overrightarrow{i^{k-1}}\right)\right\}\end{array}$ & $\begin{array}{l}\text { Approximate value for } \rho_{t}^{\overrightarrow{Z_{k}}}(\because, \mid \boldsymbol{\theta}) \text { on }\{\vec{l}\} \times[(n+1) \Delta t,(n+2) \Delta t[\times A \\
\text { Set containing all the states that step to the state }(A, \vec{l}) \text { after the } \\
(k-1) \text {-th maintenance task }\end{array}$ \\
\hline$\left(\overrightarrow{x_{L_{p}}}, \overrightarrow{y_{K_{q}}}\right)$ Mes & by fixing $\overrightarrow{\boldsymbol{D}_{O_{q}}}(t)$ to $\left(\overrightarrow{\boldsymbol{x}_{\boldsymbol{L}_{p}}}, \overrightarrow{y_{\boldsymbol{K}_{q}}}\right)$. \\
\hline
\end{tabular}

\section{INTRODUCTION}

In reliability engineering, component importance measures (IMs) are used to quantify and rank the importance of different components within a system. By determining the criticalities 
of the components, limited resources can be allocated according to components prioritization for reliability improvement during the system design and maintenance planning phases[1].

The criticality of a component changesover time,due totheevolutionof its underlying degradationprocesses[2]. Also, in practice, components are often subject to multiple competing degradation processes and any of them may individually lead to component failure[3]. The dependency among the degradation processes within one component (e.g. in a micro-engine, the shock process can enhance the wear process of rubbing surfaces and each process can lead to failure [4]) and of different components (e.g. in a water treatment plant, the decaying pre-filtrationsoften lower the performance of sand filter [5]) have to be considered in the calculation of component IMs.Moreover,the degradation processes can be interrupted by maintenance tasks(e.g.one componentcan be restored to its initial state by preventive maintenance if any of itsdegradations exceed therespective critical level[6] and by corrective maintenanceuponits failure[7]).

Neglecting the factors that influence the state of being of componentscan result in inaccurate estimation ofcomponent IMs and, thus, mislead the system designers, operatorsand managersinthe assignment of priorities to component criticalities.In this paper, we investigate the criticality of components taking into account the influence of multiple dependent competing degradation processes and maintenance tasks.

Physics-based models (PBMs) [8]and multi-state models (MSMs)[9]areused to describethe component degradation processes considered in our work.The former translatesphysics knowledge into mathematical equations thatdescribe the underlying continuous degradation processes associated to a specific mechanism,e.g. wear, corrosion andcracking [10]; the latter approximates the development ofcontinuous degradation by a process of transitions between a finite number of discrete states[11]. Recently, the authors have employedthe piecewise-deterministic Markov process (PDMP) modeling framework to incorporate PBMs and MSMs and to treat the dependency of degradation processes[12].In the present work, the authors introduce a set of PDMPs to incorporate alsomaintenance policies.

PBMs and MSMs are two widely used approaches, especially for highly reliable components, whose degradation/failure data are insufficient to build their lifetime distributions [12]. The effects of uncertain parameters in the MSMs have been considered in [13]. Global Sensitivity Analysis (GSA) has been employed to produce indices that assess the importance of the uncertain factors in the models, taking into account interactions among them. Such paper focuses on the importanceindices of uncertain factors. 
In this paper, we consider importance indices of components within multi-component systems taking into account the influence of multiple competing degradation processes, degradation dependency and maintenance tasks. GSA is not employed for such task, since it is not the uncertainty in the parameters that is considered.A literature review on component IMs is presented below, toposition our contribution within the existing works.Component IMs were first introduced mathematically by Birnbaum [14] in 1969, in a binary setting (i.e. the system and its components are either functioning or faulty). The Birnbaum IM (BIM) allows ranking components by looking at what happens to the system reliability when the reliabilities of the components are changed, one at a time. Afterwards, various IMs have been developed for binary components, including reliability achievement worth (RAW), reliability reduction worth (RRW), Fussel-Vesely and Barlow-Proschan IMs[15-17]. Other concepts of IMs have been proposed with focus to different aspects of the system, such as structure IMs, lifetime IMs, differential IMs and joint IMs [18].

For components whose description requires more than two states, e.g. to describe different degrees of functionalities or levels of degradation, definition of the component IMs have been extended in two directions: (1) metrics for components modeled by MSMs; (2) metrics for components modeled by continuous processes.Forthe first type, Armstrong [19]proposed IMs for multi-state systems (MSSs) with dual-mode failure components. For MSSs with multistate components, Griffith [20] formalized the concept of system performance based on expected utility and generalized the BIM to evaluate the effect of component improvement on system performance.Wu and Chan [21]improvedthe Griffith IM by proposinga new utility importance of a state of a component to measure which componentor which state of a certain component contributes the most to system performance.Si et al.[22] proposed the integrated IM, based on Griffith IM, to incorporate the probability distributions and transition rates of the component states, and the changes in system performance.Integrated IM can be used to evaluate how the transition of component states affects the system performance from unit time to different life stages, to system lifetime, and provide useful information for preventive actions (such as monitoring enhancement, construction improvement etc.)[23, 24]. The multistate generalized forms of classically binary IMs have been proposedby Zio and Podofillini[25]and Levitin et al.[26]:these IMsquantify the importance of a multi-state componentfor achieving a given level of performance.Ramirez-Marquez and Coit[27]developed two types of composite IMs: (1) the general composite IMsconsidering only the possible component states; (2) the alternative composite IMs considering both the 
possible component states and the associated probabilities.For the second type, Gebraeel[28]proposed a prognostics-based ranking algorithm to rank theidentical components based on their residual lives. Liu et al.[29]extended the BIM for components with multidimensional degradation processes under dynamic environments. Note that no IM has been developed for components whose (degradation) states are determined by both discrete and continuous processes, and are dependent upon other components, as it is often the case in practice [30].

To include dependency, Iyer[31] extended the Barlow-Proschan IM for components whose lifetimes are jointly absolutely continuous and possibly dependent, and Peng et al.[2] adapted the mean absolute deviation (MAD) IM (one of the alternative composite IMs) for statistically correlated (s-correlated) components subject to a one-dimension continuous degradation process; this enables to measure the expected absolute deviation in the reliability of a system with s-correlated degrading components, caused by different degrading performance levels of a particular component and the associated probabilities. To the knowledge of the authors, component IMs taking into account the dependency of multiple degradation processes within one component and among different components, with the inclusion of maintenance activities, have not been investigated in the literature(studies of IMs for repairable systems with $s$-independent components can be found in [24, 32]).

In this work, we extend the MAD to a more general settingof modeling by PDMP [33],to provide timely feedback on the criticality of a component with respect to the system reliability. The extension considers: (1) the dependency of multiple degradation processes within one component and different components; (2) discrete and continuous degradation processes; (3) two types of maintenance tasks, condition-based preventive maintenance (PM) via periodic inspections and corrective maintenance (CM).Then, a method forthe quantification of component IM is designed based on the finite-volume (FV) approach[34].

The rest of this paper is organized as follows. Section 2 presents the assumptions and degradation models under dependency and maintenance. Section 3 describes the proposed component IM. Section 4 introduces the proposed quantificationmethod. Section 5 provides a numerical example referredto one subsystem of the residual heat removal system (RHRS)[35], to demonstrate the application of the proposed component IM and feasibility of the quantification method. Finally, Section 6 concludes the work. 


\section{MODELING DEGRDATION OF UNDER DEPENDENCY MAINTENANCE PDMP}

\subsection{General assumptions}

- Consider a multi-component system, made of $Q$ components coded in the vector $\boldsymbol{O}=$ $\left\{O_{1}, O_{2}, \ldots, O_{Q}\right\}$, each one with multiple degradation processes, possibly dependent. The degradation processes can be separated into two groups: (1) $\boldsymbol{L}=\left\{L_{1}, L_{2}, \ldots\right.$, $\left.L_{M}\right\}$ modeled by $M$ PBMs; (2) $\boldsymbol{K}=\left\{K_{1}, K_{2}, \ldots, K_{N}\right\}$ modeled by NMSMs, where $L_{m}, m=1,2, \ldots, M$ and $K_{n}, n=1,2, \ldots, N$ are the indicesof the degradation processes.

- The degradation state of a component $O_{q} \in \boldsymbol{O}, q=1,2, \ldots, Q$, is determined by its degradation processes $\boldsymbol{D}_{O_{q}} \subseteq \boldsymbol{L} \cup \boldsymbol{K}$ and the component fails either when one of the degradation processes evolves beyond a threshold of failure in the continuous state stochastic processor reaches the discrete failure statein the multi-state stochastic transition process.

- A degradation process $L_{m} \in \boldsymbol{L}$ in the first groupisdescribed by $d_{L_{m}}$ time-dependent continuous $\quad$ variables $\quad \overrightarrow{X_{L_{m}}}(t)=\left(\overrightarrow{X_{L_{m}}^{\boldsymbol{P}}}(t), \overrightarrow{X_{L_{m}}^{\boldsymbol{P}}}(t)\right) \in \mathbb{R}^{d_{L_{m}}} \quad, \quad$ whose evolutionsaredescribed by a set of first-order differential equations (physics equations) in terms of: (1) the non-decreasing degradation variables vector $\overrightarrow{X_{L_{m}}^{D}}(t)$ (e.g. crack length)representing the component degradation condition; (2) the physical variables vector $\overrightarrow{X_{L_{m}}^{\boldsymbol{P}}}(t)$ (e.g. velocity) influencing $\overrightarrow{X_{L_{m}}^{\boldsymbol{D}}}(t)$ and vice versa. Due to degradation process $L_{m}$, the component fails when any degradation variable $x_{L_{m}}^{i}(t) \in \overrightarrow{X_{L_{m}}^{D}}(t)$ exceeds its corresponding failure threshold denoted by $x_{L_{m}}^{i}{ }^{*}$. The set of failure states of the degradation variables $\overrightarrow{X_{L_{m}}}(t)$ is denoted by $\mathcal{F}_{L_{m}}$.

- A degradation process $K_{n} \in \boldsymbol{K}$ in the second group is described by the state variable $Y_{K_{n}}(t)$, which takes values from a finite state set $\boldsymbol{S}_{K_{n}}=\left\{0_{K_{n}}, 1_{K_{n}}, \ldots, d_{K_{n}}\right\}$, where ' $d_{K_{n}}$ ' is the perfect functioning state and ' $0_{K_{n}}$ ' is the complete failure state. All intermediate states are functioning or partially functioning. The evolution of the degradation process is characterized by the transition rates between states. The failure state set of the multi-state stochastic transition process of degradation $Y_{K_{n}}(t)$ is described by $\mathcal{F}_{K_{n}}=\left\{0_{K_{n}}\right\}$. 
- Dependencies between degradation processes may existboth within and between groups $\boldsymbol{L}$ and $\boldsymbol{K}$. The detailed formulations are given in eqs. (1-3).

- For degradation process $i \in \boldsymbol{L} \cup \boldsymbol{K}$, the inspection task $I_{i}$ of PM is performed with fixed period $T_{i}$ and bringsthe related component back to its initial statewheniis found in the predefined state set $\boldsymbol{H}_{i}$.

- The component is restored to its initial state by CM, as soon as it fails.

- The inspection tasks and all maintenance actions are done instantaneously and without errors.

An illustration of two components $O_{1}$ and $O_{2}$ is shown in Fig. 1, where $\boldsymbol{D}_{O_{1}}=\left\{L_{1}\right\}$ and $\boldsymbol{D}_{O_{2}}=\left\{K_{1}\right\} . \mathrm{PM}$ is performed for $L_{1}$ if $\overrightarrow{X_{L_{1}}^{\boldsymbol{D}}}(t)$ exceeds its threshold $x_{L_{1}}{ }^{p}$ at the time of inspection and for $K_{1}$ if $Y_{K_{1}}(t)$ is in state 1 at the time of inspection.

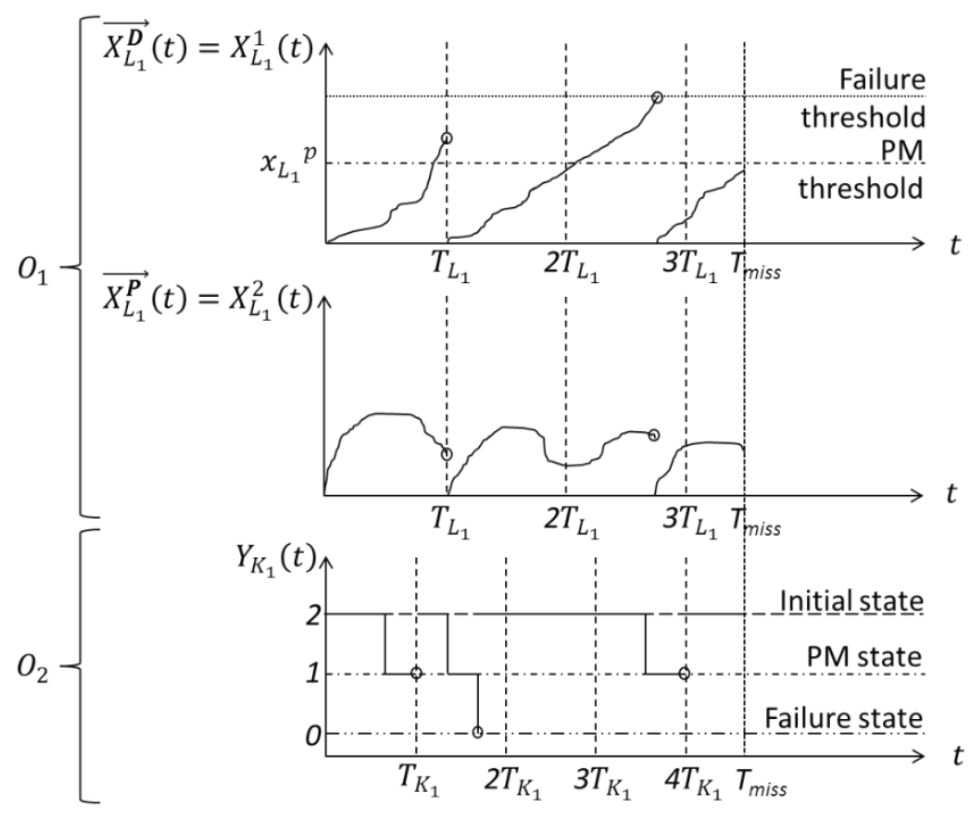

Fig. 1. An illustration of two components.

\subsection{Degradation model of the system}

The degradation state of the system is represented as 


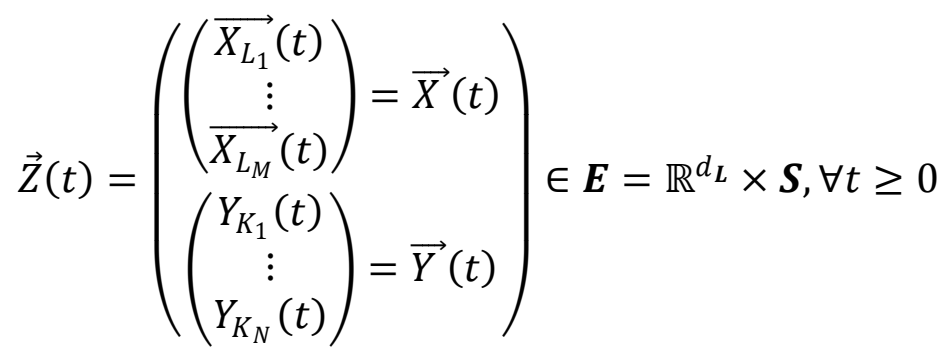

where $E$ is the space combining $\mathbb{R}^{d_{\boldsymbol{L}}}\left(d_{\boldsymbol{L}}=\sum_{m=1}^{M} d_{L_{m}}\right)$ and $\boldsymbol{S}\left(\boldsymbol{S}=\prod_{n=1}^{N} \boldsymbol{S}_{K_{\boldsymbol{n}}}\right)$.

A setof PDMPs $\overrightarrow{Z_{k}}(t), k=1,2, \ldots$ is employed to model the system degradation processes, where a new PDMP is established once a maintenance task is performed.Let $N_{m}$ denote the total number of maintenance tasks (PM and $\mathrm{CM}$ ) the system has experienced till the mission time $T_{\text {miss }}$, then, $\overrightarrow{Z_{k}}(t), k=1,2, \ldots, N_{m}$ is defined on $\left[T_{m}^{k-1}, T_{m}^{k}\right]$, where $T_{m}^{k}, k=1,2, \ldots, N_{m}$ denotes the execution time of the $k$-th maintenance task and $T_{m}^{0}=0 . \overrightarrow{Z_{N_{m}+1}}(t)$ is defined on $\left[T_{m}^{N_{m}}, T_{m i s s}\right]$.Fig. 2 shows this for the degradation processes in Fig. 1.

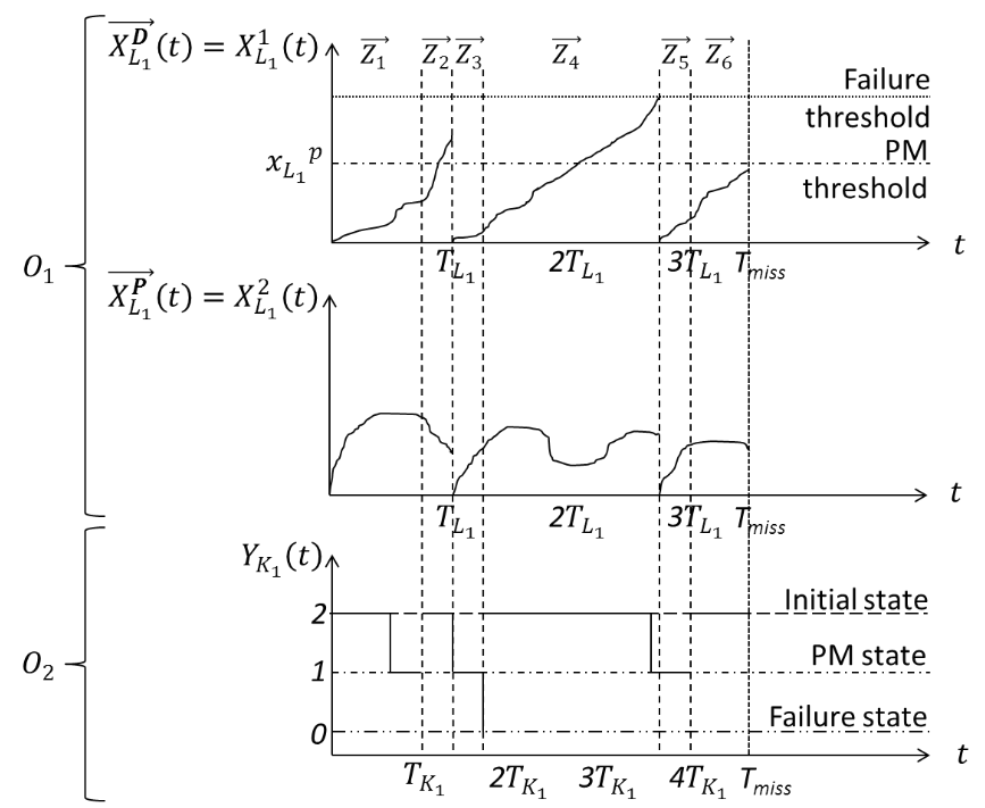

Fig. 2. An illustration of two components, modeled byPDMPs.

The evolution of the elements $\overrightarrow{Z_{k}}(t), k=1,2, \ldots, N_{m}+1$, of the system state vector $\vec{Z}(t)$ involves (1) the stochastic transition process of $\vec{Y}(t)$ and (2) the deterministic progression of $\vec{X}(t)$, between successive transitions of $\vec{Y}(t)$, given $\vec{Y}(t)$. The first process is governed by the transition ratesof $\vec{Y}(t)$ : 


$$
\begin{aligned}
\lim _{\Delta t \rightarrow 0} P\left(\vec{Y}(t+\Delta t)=\vec{j} \mid \overrightarrow{Z_{k}}(t)=(\vec{X}\right. & \left.(t), \vec{Y}(t)=\vec{\imath})^{T}, \boldsymbol{\theta}_{\boldsymbol{K}}\right) \\
& =\lambda_{\vec{i}}\left(\vec{\jmath} \mid \vec{X}(t), \boldsymbol{\theta}_{\boldsymbol{K}}\right) \Delta t, \forall \vec{\imath}, \vec{\jmath} \in \boldsymbol{S}, \vec{\imath} \neq \vec{\jmath}(2)
\end{aligned}
$$

wherethe parameter vector $\boldsymbol{\theta}_{\boldsymbol{K}}$ represents environmental and operational factorsinfluencing the degradation processes in $\boldsymbol{K}$, and $\lambda_{\vec{i}}\left(\vec{\jmath} \mid \vec{X}(t), \boldsymbol{\theta}_{\boldsymbol{K}}\right)$ is the transition rate from state $\vec{\imath}$ to $\vec{\jmath}$. The second evolution processis described by the deterministic physics equations as follows:

$$
\overrightarrow{\dot{X}}(t)=\left(\begin{array}{c}
\overrightarrow{X_{L_{1}}}(t) \\
\vdots \\
\overrightarrow{X_{L_{M}}}(t)
\end{array}\right)=\left(\begin{array}{c}
\overrightarrow{f_{L_{1}}}\left(\overrightarrow{Z_{k}}(t), t \mid \boldsymbol{\theta}_{L_{1}}\right) \\
\vdots \\
\left.\overrightarrow{{\overrightarrow{L_{M}}}_{\left(Z_{k}\right.}}(t), t \mid \boldsymbol{\theta}_{L_{M}}\right)
\end{array}\right)=\overrightarrow{f_{L}}\left(\overrightarrow{Z_{k}}(t), t \mid \boldsymbol{\theta}_{\boldsymbol{L}}=\left(\boldsymbol{\theta}_{L_{1}}, \boldsymbol{\theta}_{L_{2}}, \ldots, \boldsymbol{\theta}_{L_{M}}\right)\right)(3)
$$

wherethe parameter vector $\boldsymbol{\theta}_{L_{m}}, m=1,2, \ldots, M$ represents environmental and operational factors influencing the degradation processes in $L_{m} \cdot \overrightarrow{Z_{k}}\left(T_{m}^{k-1}\right)$ (the initial states of $\overrightarrow{Z_{k}}(t), k=$ $\left.2, \ldots, N_{m}+1\right)$ can be obtained according to $\overrightarrow{Z_{k-1}}\left(T_{m}^{k-1}\right)$ and the $(k-1)$-th maintenance task. The degradation states of the system till $T_{\text {miss }}$ can be represented by

$$
\vec{Z}(t)=\sum_{k=1}^{N_{m}} \mathbf{1}_{\left[T_{m}^{k-1}, T_{m}^{k}[\right.}(t) \cdot \overrightarrow{Z_{k}}(t)+\mathbf{1}_{\left[T_{m}^{N_{m}}, T_{m i s s}\right]}(t) \cdot \overrightarrow{Z_{N_{m}+1}}(t)(4)
$$

Since maintenance is performed instantaneously, the failure states of the system are infinitely approachable by $\vec{Z}(t)$, instead of being truly reached. We, then, use another stochastic process $\vec{Z}(t)$, which canrecord the failure of the system as follows:

$$
\overrightarrow{Z^{\prime}}(t)=\mathbf{1}_{\left[0, T_{m}^{1}\right]}(t) \cdot \overrightarrow{Z_{1}}(t)+\sum_{k=2}^{N_{m}} \mathbf{1}_{] T_{m}^{k-1}, T_{m}^{k}\right]} \cdot \overrightarrow{Z_{k}}(t)+\mathbf{1}_{\left.T_{m}^{N_{m}}, T_{m i s s}\right]}(t) \cdot \overrightarrow{Z_{N_{m}+1}}(t)(5)
$$

Let $\mathcal{F}$ denote the system failure state set: then, the system reliability at $T_{\text {miss }}$ can bedefined as follows:

$$
R\left(T_{\text {miss }}\right)=P\left[\overrightarrow{Z^{\prime}}(s) \notin \mathcal{F}, \forall s \leq T_{\text {miss }}\right]=P\left[\cap _ { k = 1 } ^ { N _ { m } } ( \vec { Z _ { k } } ( T _ { m } ^ { k } ) \notin \mathcal { F } ) \cap \left(\overrightarrow{Z_{N_{m}+1}}\left(T_{\text {miss }}\right) \notin\right.\right.
$$

Since the component is restored to its initial state by corrective maintenance as soon as it fails, the failure states of the system can only be reached by $\vec{Z}(t)$ at the execution time of the maintenance tasks $T_{m}^{k}, k=1,2, \ldots, N_{m}$ or at the mission time $T_{\text {miss }}$. Therefore, the event $\overrightarrow{Z^{\prime}}(s) \notin \mathcal{F}, \forall s \leq T_{\text {miss }}$ can be represented by $\bigcap_{k=1}^{N_{m}}\left(\overrightarrow{Z_{k}}\left(T_{m}^{k}\right) \notin \mathcal{F}\right) \cap\left(\overrightarrow{Z_{N_{m}+1}}\left(T_{m i s s}\right) \notin \mathcal{F}\right)$.

\section{COMPONENT IM}

Ramirez-Marquez and Coit[27]proposed the MAD IM for MSSs with multi-state components, which evaluates the components criticality taking into account allthe possible states and associated probabilities. Peng et al.[2]adaptedit for binary systems with $s$-correlated components subject to one continuous degradation process. 
For components whose (degradation) states are determined by both discrete and continuous processes, we proposean extension of MAD to provide timely feedbacks of thecriticality of component $O_{q}$ with multiple dependent competing degradation processes modeled by MSMs and PBMs, and giving consideration toPM and CM. The formulation is presentedas follows:

$$
C I_{O_{q}}(t)=E\left[\left|P\left(\overrightarrow{Z^{\prime}}(s) \notin \mathcal{F}, \forall s \leq t \mid \overrightarrow{\boldsymbol{D}_{O_{q}}}(t)\right)-R(t)\right|\right](7)
$$

where $\overrightarrow{\boldsymbol{D}_{O_{q}}}(t)=\left(\overrightarrow{X_{L_{p}}}(t)=\left(\overrightarrow{X_{L_{p_{1}}}}(t), \ldots, \overrightarrow{X_{L_{p_{n}}}}(t)\right), \overrightarrow{Y_{\boldsymbol{K}_{q}}}(t)=\left(Y_{K_{q_{1}}}(t), \ldots, Y_{K_{q_{m}}}(t)\right)\right)$ and $\boldsymbol{D}_{O_{q}}=$ $\left\{\boldsymbol{L}_{p}=\left\{L_{p_{1}}, \ldots, L_{p_{n}}\right\}, \boldsymbol{K}_{q}=\left\{K_{q_{1}}, \ldots, K_{q_{m}}\right\}\right\}$.It accounts for the expected absolute deviation in the system reliability caused by changes of all degradation processes of component $O_{q}$. Let $\mathbb{R}^{d_{\boldsymbol{L}_{p}}}=\mathbb{R}^{\sum_{i=1}^{n} d_{L_{p_{i}}}}$ and $\boldsymbol{S}_{\boldsymbol{K}_{q}}=\prod_{i=1}^{m} \boldsymbol{S}_{K_{q_{i}}}$ denote the state space of $\overrightarrow{X_{\boldsymbol{L}_{p}}}(t)$ and $\overrightarrow{Y_{\boldsymbol{K}_{q}}}(t)$, respectively;eq. (7) can, then, be expressed as

$$
\begin{aligned}
& C I_{O_{q}}(t)= \sum_{\overrightarrow{y_{\boldsymbol{K}_{q}}} \in \boldsymbol{S}_{\boldsymbol{K}_{q}}} \int_{\overrightarrow{\bar{x}_{\boldsymbol{L}_{p}}} \in \mathbb{R}^{d}} f_{\overrightarrow{\boldsymbol{L}_{p}}} \overrightarrow{\boldsymbol{D}_{O_{q}}}(t) \\
&\left|P\left(\overrightarrow{Z^{\prime}}(s) \notin \mathcal{F}, \forall s \leq t \mid \overrightarrow{X_{\boldsymbol{L}_{p}}}(t)=\overrightarrow{\boldsymbol{L}_{p}}, \overrightarrow{y_{\boldsymbol{K}_{q}}}, \overrightarrow{Y_{\boldsymbol{K}_{q}}}(t)=\overrightarrow{y_{\boldsymbol{K}_{q}}}\right)-R(t)\right|
\end{aligned}
$$

where $f_{\overrightarrow{D_{O_{q}}}(t)}\left(d \overrightarrow{x_{\boldsymbol{L}_{p}}}, \overrightarrow{y_{\boldsymbol{K}_{q}}}\right)$ is theprobability distributionof $\overrightarrow{\boldsymbol{D}_{O_{q}}}(t)$.

Let $N_{m}^{t} \geq 1$ denote the number of maintenance tasks that the system has experienced till $t$. According to eq. (6), we can obtain that:

$$
R\left(T_{\text {miss }}\right)=P\left[\left(\bigcap_{k=1}^{N_{m}^{t}}\left(\overrightarrow{Z_{k}}\left(T_{m}^{k}\right) \notin \mathcal{F}\right)\right) \cap\left(\overrightarrow{Z_{N_{m}^{t}+1}}(t) \notin \mathcal{F}\right)\right]
$$

and

$$
\begin{aligned}
& P\left(\overrightarrow{Z^{\prime}}(s) \notin \mathcal{F}, \forall s \leq t \mid \overrightarrow{X_{L_{p}}}(t)=\overrightarrow{x_{L_{p}}}, \overrightarrow{Y_{\boldsymbol{K}_{q}}}(t)=\overrightarrow{y_{\boldsymbol{K}_{q}}}\right)=
\end{aligned}
$$

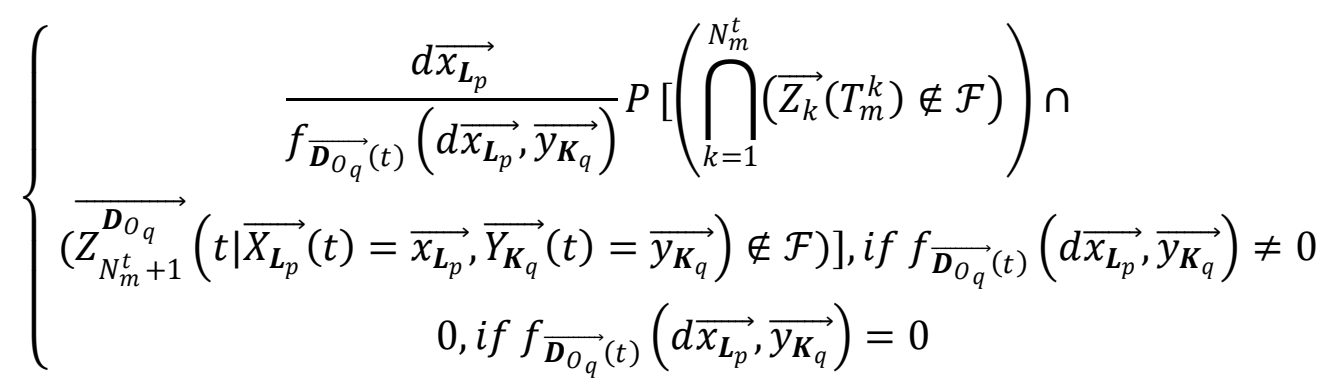

$$
\begin{aligned}
& \text { where } \overrightarrow{Z_{N_{m}+1}^{D_{O_{q}}}}\left(t \mid \overrightarrow{X_{\boldsymbol{L}_{p}}}(t)=\overrightarrow{x_{\boldsymbol{L}_{p}}}, \overrightarrow{Y_{\boldsymbol{K}_{q}}}(t)=\overrightarrow{y_{\boldsymbol{K}_{q}}}\right)=\left(\overrightarrow{X_{L_{1}}}(t), \ldots, \overrightarrow{X_{\boldsymbol{L}_{p}}}(t)=\overrightarrow{x_{\boldsymbol{L}_{p}}}, \ldots, \overrightarrow{X_{L_{M}}}(t), Y_{K_{1}}(t),\right. \\
& \left.\ldots, \overrightarrow{Y_{\boldsymbol{K}_{q}}}(t)=\overrightarrow{y_{\boldsymbol{K}_{q}}}, \ldots, Y_{K_{N}}(t)\right)^{T} .
\end{aligned}
$$




\section{FV SCHEME FOR COMPONENT IM QUANTIFICATION}

Let $\overrightarrow{p_{t}}\left(d \vec{x}, \vec{\imath} \mid \boldsymbol{\theta}=\boldsymbol{\theta}_{\boldsymbol{L}} \cup \boldsymbol{\theta}_{\boldsymbol{K}}\right), \forall \vec{x} \in \mathbb{R}^{d_{\boldsymbol{L}}}, \vec{\imath} \in \boldsymbol{S}$ denote the probability distribution of processes $\overrightarrow{Z_{k}}(t)$. Due to the complex behavior of the PDMP, the analytical solution for theprobability distribution is difficult to obtain [36]. TheFV approachdeveloped in [34]can be used to obtain the approximated solution by discretizing the time space and the state space of the continuous variables, achievingaccurate results within an admissible computing time,as shown in [37].

\subsection{FV scheme for PDMP}

\subsubsection{Assumptions}

This approach can be applied under the following assumptions:

- $\quad \lambda_{\vec{i}}\left(\vec{j}, \mid \boldsymbol{\theta}_{\boldsymbol{K}}\right), \forall \vec{\imath}, \vec{j} \in \boldsymbol{S}$ are continuous and bounded functions from $\mathbb{R}^{d_{\boldsymbol{L}}}$ to $\mathbb{R}^{+}$.

- $\overrightarrow{f_{L}}\left(\because, \cdot \mid \boldsymbol{\theta}_{L}\right), \forall \vec{\imath} \in S$ are continuous functions from $\mathbb{R}^{d_{L}} \times \mathbb{R}^{+}$to $\mathbb{R}^{d_{L}}$ and locally Lipschitz continuous.

- $\overrightarrow{f_{L}}\left(\cdot, t \mid \boldsymbol{\theta}_{L}\right), \forall \vec{\imath} \in \boldsymbol{S}$ are sub-linear, i.e. there are some $V_{1}>0$ and $V_{2}>0$ such that

$$
\forall \vec{x} \in \mathbb{R}^{d_{L}}, t \in \mathbb{R}^{+}\left|\overrightarrow{f_{L}^{\vec{l}}}\left(\vec{x}, t \mid \boldsymbol{\theta}_{L}\right)\right| \leq V_{1}(\|\vec{x}\|+|t|)+V_{2}
$$

- $\quad \operatorname{div}\left(\overrightarrow{f_{L}}\left(\cdot, \cdot \mid \boldsymbol{\theta}_{\boldsymbol{L}}\right)\right), \forall \vec{\imath} \in \boldsymbol{S}$ are almost everywhere bounded in absolute value by some real value $D>0$ (independent of $\vec{\imath}$ ).

\subsubsection{Solution approach}

The time space $\mathbb{R}^{+}$is divided into small intervals $\mathbb{R}^{+}=\bigcup_{n=0,1,2, \ldots}[n \Delta t,(n+1) \Delta t[$ by setting the length of each interval $\Delta t>0$ and the state space $\mathbb{R}^{d_{L}}$ of $\vec{X}(t)$ is divided into an admissible mesh $\mathcal{M}$ which satisfies that:

(1) $\bigcup_{A \in \mathcal{M}} A=\mathbb{R}^{d_{L}}$.

(2) $\forall A, B \in \mathcal{M}, A \neq B \Rightarrow A \cap B=\emptyset$.

(3) $m_{A}=\int_{A} \overrightarrow{d x}>0, \forall A \in \mathcal{M}$, where $m_{A}$ is the volume of grid $A$.

(4) $\sup _{A \in \mathcal{M}} \operatorname{diam}(A)<+\infty$ where $\operatorname{diam}(A)=\sup _{\forall \vec{x}, \vec{y} \in A}|\vec{x}-\vec{y}|$. 
The numerical scheme aims at constructing an approximate value $\overrightarrow{\rho_{t}}(\vec{x}, \mid \boldsymbol{\theta}) d \vec{x}$ for $p_{t}^{\overrightarrow{Z_{k}}}(d \vec{x}, \cdot \mid \boldsymbol{\theta})$, such that $\overrightarrow{\rho_{t}^{Z_{k}}}(\vec{x}, \cdot \mid \boldsymbol{\theta})$ is constant on each $A \times\{\vec{\imath}\} \times[n \Delta t,(n+1) \Delta t[, \forall A \in$ $\mathcal{M}, \vec{\imath} \in S,\left[n \Delta t,(n+1) \Delta t\left[\in\left[T_{m}^{k-1}, T_{m}^{k}\right]:\right.\right.$

$$
\rho_{t}^{\overrightarrow{Z_{k}}}(\vec{x}, \vec{\imath} \mid \boldsymbol{\theta})=P_{n}^{\overrightarrow{Z_{k}}}(A, \vec{\imath} \mid \boldsymbol{\theta}), \forall \vec{\imath} \in \boldsymbol{S}, \vec{x} \in A, t \in[n \Delta t,(n+1) \Delta t[
$$

$P_{0}^{\overrightarrow{Z_{k}}}(A, \vec{\imath} \mid \boldsymbol{\theta}), \forall \vec{\imath} \in \boldsymbol{S}, A \in \mathcal{M}$ is defined as follows:

$$
P_{0}^{\overrightarrow{Z_{k}}}(A, \vec{\imath} \mid \boldsymbol{\theta})=\int_{A} p_{0}^{\overrightarrow{Z_{k}}}(d \vec{x}, \vec{\imath} \mid \boldsymbol{\theta}) / m_{A}
$$

Then, $P_{n+1}^{\overrightarrow{Z_{k}}}(A, \vec{\imath} \mid \boldsymbol{\theta}), \forall \vec{\imath} \in S, A \in \mathcal{M}, n \in \mathbb{N}$ can be calculated considering the deterministic evaluation of $\vec{X}(t)$ and the stochastic evolution of $\vec{Y}(t)$ based on $P_{n}^{\overrightarrow{Z_{k}}}(\mathcal{M}, \vec{l} \mid \boldsymbol{\theta})$ by the Chapman-Kolmogorov forward equation[38], as follows:

$$
\begin{gathered}
P_{n+1}^{\overrightarrow{Z_{k}}}(A, \vec{\imath} \mid \boldsymbol{\theta}) \\
=\frac{1}{1+\Delta t b_{A}^{\vec{i}}} \widehat{\overrightarrow{P_{n+1}^{Z_{k}}}}(A, \vec{\imath} \mid \boldsymbol{\theta})+\Delta t \sum_{\vec{j} \in S} \frac{a_{A}^{\vec{j}}}{1+\Delta t b_{A}^{\vec{\jmath}}} \widehat{\overrightarrow{P_{n}}}(A, \vec{\jmath} \mid \boldsymbol{\theta})
\end{gathered}
$$

where

$$
a_{A}^{\vec{j} \vec{l}}=\int_{A} \lambda_{\vec{j}}\left(\vec{\imath}, \vec{x} \mid \boldsymbol{\theta}_{K}\right) \overrightarrow{d x} / m_{A}, \forall \vec{\imath} \in \boldsymbol{S}, A \in \mathcal{M}
$$

is the average transition rate from state $\vec{\jmath}$ to state $\vec{\imath}$ for grid $A$,

$$
b_{A}^{\vec{i}}=\sum_{\vec{j} \neq \vec{\imath}} a_{A}^{\vec{i} \vec{j}}, \forall \vec{\imath} \in S, A \in \mathcal{M}
$$

is the average transition rate out of state $\vec{\imath}$ for grid $A$,

$$
\widehat{P_{n+1}^{\overline{Z_{k}}}}(A, \vec{\imath} \mid \boldsymbol{\theta})=\sum_{B \in \mathcal{M}} m_{B A}^{\vec{\imath}} P_{n}^{\overline{Z_{k}}}(B, \vec{\imath} \mid \boldsymbol{\theta}) / m_{A}, \forall \vec{\imath} \in \boldsymbol{S}, A \in \mathcal{M}
$$

is the approximate value of probability density function on $\{\vec{l}\} \times[(n+1) \Delta t,(n+2) \Delta t[\times A$ according to the deterministic evaluation of $\vec{X}(t)$,

$$
m_{B A}^{\vec{\imath}}=\int_{\{\vec{y} \in B} \overrightarrow{\left.\mid g^{\vec{i}}\left(\vec{y}, \Delta t \mid \boldsymbol{\theta}_{L}\right) \in A\right\}}, \overrightarrow{d y}, \forall \vec{\imath} \in S, A, B \in \mathcal{M}
$$

is the volume of the part of grid $B$ which will enter grid $A$ after time $\Delta t$ according to the deterministic evaluation of $\vec{X}(t)$, where $\overrightarrow{g^{i}}(\because): \mathbb{R}^{d_{L}} \times \mathbb{R} \rightarrow \mathbb{R}^{d_{L}}$ is the solution of

$$
\frac{\partial}{\partial t} \overrightarrow{g^{i}}\left(\vec{y}, t \mid \boldsymbol{\theta}_{\boldsymbol{L}}\right)=\overrightarrow{f_{L}}\left(\overrightarrow{g^{i}}\left(\vec{y}, t \mid \boldsymbol{\theta}_{\boldsymbol{L}}\right), t \mid \boldsymbol{\theta}_{\boldsymbol{L}}\right)(
$$

with

$$
\overrightarrow{g^{i}}\left(\vec{y}, 0 \mid \boldsymbol{\theta}_{L}\right)=\vec{y}
$$

$\overrightarrow{g^{i}}\left(\vec{y}, \Delta t \mid \boldsymbol{\theta}_{L}\right)$ gives the state of the deterministic behavior of $\vec{X}(t)$ after time $\Delta t$, starting from the state $\vec{y}$ while the processes $\vec{Y}(t)$ stay in state $\vec{\imath}$. 


\subsection{Quantification of component IM}

Given the initial probability distribution $p_{0}^{\overrightarrow{Z_{1}}}(d \vec{x}, \vec{\imath} \mid \boldsymbol{\theta})$ of the system, $P_{0}^{\overrightarrow{Z_{1}}}(A, \vec{\imath} \mid \boldsymbol{\theta}), \forall \vec{\imath} \in$ $\boldsymbol{S}, A \in \mathcal{M}$, can be obtained as:

$$
P_{0}^{\overrightarrow{Z_{1}}}(A, \vec{\imath} \mid \boldsymbol{\theta})=\int_{A} p_{0}^{\overrightarrow{Z_{1}}}(d \vec{x}, \vec{\imath} \mid \boldsymbol{\theta}) / m_{A}
$$

$P_{\left.\mid T_{m}^{1} / \Delta t\right\rceil}^{\overrightarrow{Z_{1}}}(A, \vec{\imath} \mid \boldsymbol{\theta}), \forall \vec{\imath} \in \boldsymbol{S}, A \in \mathcal{M}$ can, then, be calculated through the FV scheme.

To calculate eq.(9) and $P\left[\left(\bigcap_{k=1}^{N_{m}^{t}}\left(\overrightarrow{Z_{k}}\left(T_{m}^{k}\right) \notin \mathcal{F}\right)\right) \cap \overrightarrow{Z_{N_{m}^{t}+1}^{\boldsymbol{D}_{O_{q}}}}\left(t \mid \overrightarrow{X_{\boldsymbol{L}_{p}}}(t)=\overrightarrow{\boldsymbol{X}_{\boldsymbol{L}_{p}}}, \overrightarrow{Y_{\boldsymbol{K}_{q}}}(t)=\right.\right.$ $\left.\left.\left.\overrightarrow{y_{K_{q}}}\right) \notin \mathcal{F}\right)\right]$ in eq. (10), we are only interested in the situation that the system is functioning till $t$; thus, $P_{\left.\mid T_{m}^{k-1} / \Delta t\right\rceil}^{\overrightarrow{Z_{k}}}(A, \vec{\imath} \mid \boldsymbol{\theta}), \forall \vec{\imath} \in S, A \in \mathcal{M}, k=2,3, \ldots N_{m}^{t}+1$ is initiated as follows:

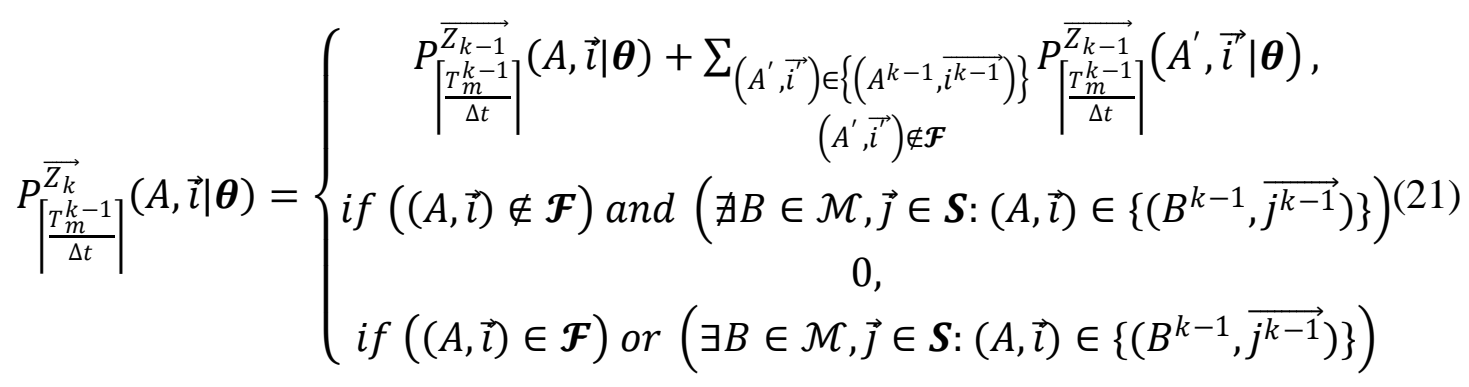

where $\left\{\left(A^{k-1}, \overrightarrow{i^{k-1}}\right)\right\}, \forall \vec{\imath} \in S, A \in \mathcal{M}$, is the set containing all the states that step to the state $(A, \vec{\imath})$ caused by the $(k-1)$-th maintenance task. Then, we can obtain that

$$
\begin{aligned}
& P\left[\left(\bigcap_{k=1}^{N_{m}^{t}}\left(\overrightarrow{Z_{k}}\left(T_{m}^{k}\right) \notin \mathcal{F}\right)\right) \cap\left(\overrightarrow{Z_{N_{m}^{t}+1}}(t) \notin \mathcal{F}\right)\right]=\sum_{(A, \vec{\imath}) \notin \mathcal{F}} m_{A} P_{\left[\frac{t}{\Delta t}\right]}^{\overrightarrow{Z_{m}^{t}+1}}(A, \vec{\imath} \mid \boldsymbol{\theta}) \\
& P\left[\left(\bigcap_{k=1}^{N_{m}^{t}}\left(\overrightarrow{Z_{k}}\left(T_{m}^{k}\right) \notin \mathcal{F}\right)\right) \cap\left(\overrightarrow{Z_{N_{m}^{t}+1}^{D_{O_{q}}}}\left(t \mid \overrightarrow{X_{\boldsymbol{L}_{p}}}(t)=\overrightarrow{{\boldsymbol{L}_{\boldsymbol{L}}}_{p}}, \overrightarrow{Y_{\boldsymbol{K}_{q}}}(t)=\overrightarrow{y_{\boldsymbol{K}_{q}}}\right) \notin \mathcal{F}\right)\right]=
\end{aligned}
$$

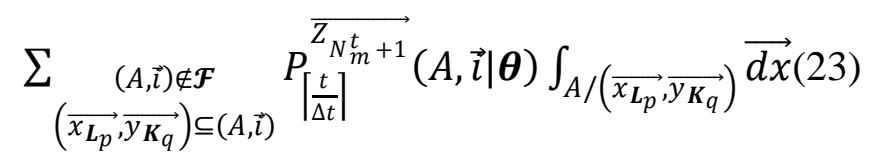

where $A /\left(\overrightarrow{x_{\boldsymbol{L}_{p}}}, \overrightarrow{y_{\boldsymbol{K}_{q}}}\right)$ is the mesh by fixing $\overrightarrow{\boldsymbol{D}_{O_{q}}}(t)$ to $\left(\overrightarrow{\boldsymbol{x}_{\boldsymbol{L}_{p}}}, \overrightarrow{y_{\boldsymbol{K}_{q}}}\right)$.

To calculate $f_{\overrightarrow{D_{O_{q}}}}(t)\left(d \overrightarrow{\boldsymbol{x}_{\boldsymbol{L}_{p}}}, \overrightarrow{y_{\boldsymbol{K}_{q}}}\right)$ in eq. (8), (10), we are interested in the state of the systemat tno matter whether the system is functioning tillt or not; thus, $P_{\left[T_{m}^{k-1} / \Delta t\right\rceil}^{\overrightarrow{Z_{k}}}(A, \vec{\imath} \mid \boldsymbol{\theta}), \forall \vec{\imath} \in \boldsymbol{S}, A \in$ $\mathcal{M}, k=2,3, \ldots N_{m}^{t}+1$ is initiated as follows: 


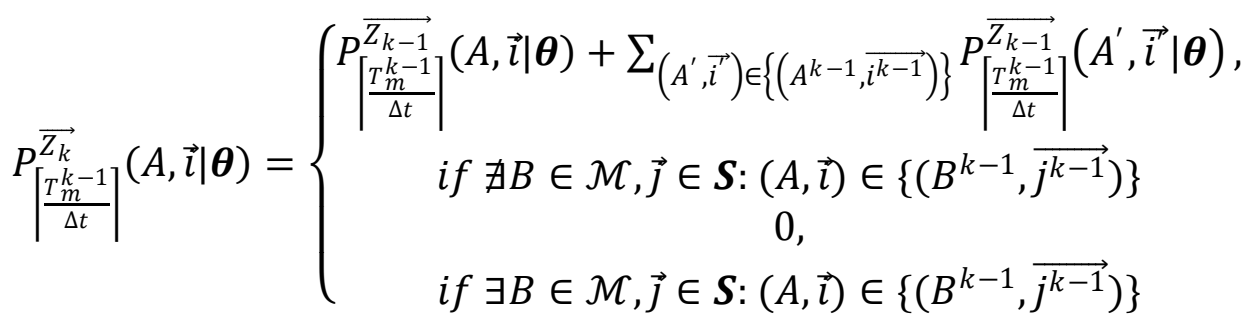

We can obtain that

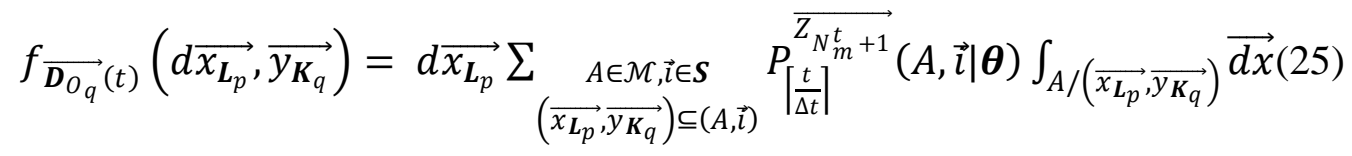

$C I_{O_{q}}(t)$ can, then, be obtainedbyusingeqs. (8)-(10), (20)-(25).

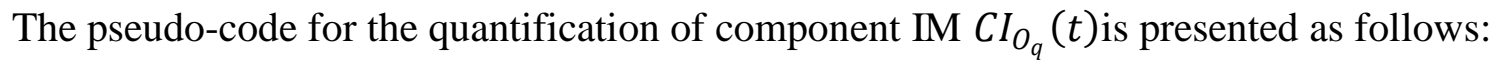

Set timet, length of each interval $\Delta t$ and admissible mesh $\mathcal{M}$

Set the initial probability distribution $\overrightarrow{p_{0}}(d \vec{x}, \vec{\imath} \mid \boldsymbol{\theta})$

Initialize the probability distribution of $\overrightarrow{Z_{1}}(0)$ by using eq. (20)

$$
\text { For } j=1 \text { to } N_{m}^{t} \text { do }
$$

Calculate theprobability distribution of $\overrightarrow{Z_{j}}\left(T_{m}^{j}\right)$ by using FV scheme

Calculate the initial probability distribution of $\overrightarrow{Z_{j+1}}\left(T_{m}^{j}\right)$ by using eq. (21)

\section{End}

Calculate the probability distribution of $\overrightarrow{Z_{N_{m}^{t}+1}}(t)$ by using FV scheme

Calculate the system reliability at time tby using eq. (22)

Calculate the conditional system reliability at time tby using eq. (23)

For $j=1$ to $N_{m}^{t}$ do

Calculate the probability distribution of $\vec{Z}_{j}\left(T_{m}^{j}\right)$ by using FV scheme

Calculate the initial probability distribution of $\overrightarrow{Z_{j+1}}\left(T_{m}^{j}\right)$ by using eq. (24)

End 
Calculate the probability distribution of $\overrightarrow{Z_{N_{m}^{t}+1}}(t)$ by using FV scheme

Calculate the probability distribution of $\overrightarrow{\boldsymbol{D}_{O_{q}}}(t)$ by using eq. (25)

Calculate the component IM $C I_{O_{q}}(t)$ by using eq. (8)

\section{ILLUSTRATIVE CASE}

The system consists of a centrifugal pump and a pneumatic valve in series, and is asubsystem of the residual heat removal system (RHRS) of a nuclear power plant of Électricite de France (EDF). Given the series configuration, the failure of anyone of the twocomponentscan lead the subsystem to failure. A dependency in the degradation processesof the two components has been indicated by the experts: the pump vibrates due to degradation[39]which, in turn, leads the valve to vibrate, aggravatingits own degradation processes [40].

\subsection{Centrifugal pump}

The pump is modeled by a MSM, modified from the one originally supplied by EDFupon discussion with the experts. It is a continuous-time homogeneous Markov chain as shown in Fig.3:

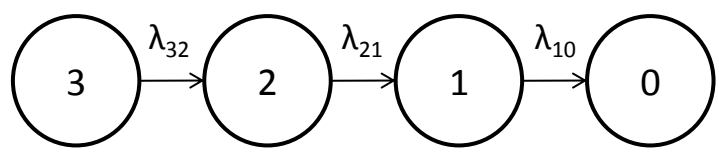

Fig. 3. Degradation process of the pump.

$S_{p}=\{0,1,2,3\}$ denotesits degradation states set, where 3 is the perfect functioning state and 0 is the complete failure state. The parameters $\lambda_{32}, \lambda_{21}$ and $\lambda_{10}$ are the transition rates between the degradation states.Due to degradation,the pump vibrates when it reaches the degradation states 2 and 1 . The intensity of the vibration of the pump on states 2 and 1 is evaluated as by the experts 'smooth' and 'rough', respectively. 


\subsection{Pneumatic valve}

The simplified scheme of the pneumatic valveis shown in Fig.4. It is a normally-closed, gas-actuated valve with a linear cylinder actuator.

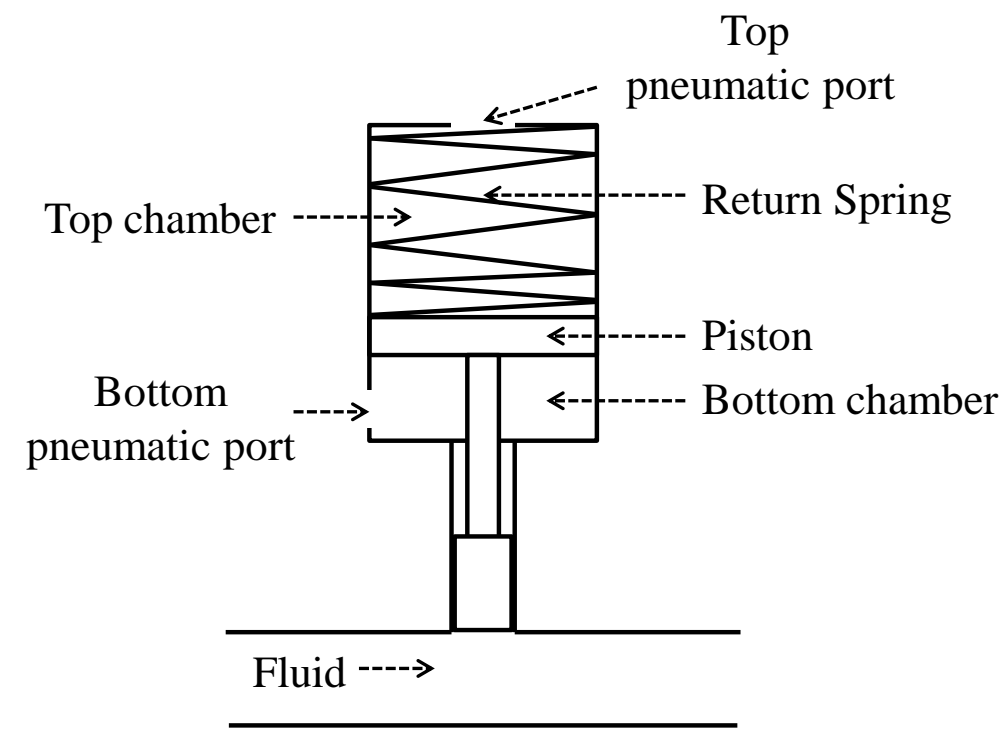

Fig. 4. Simplified scheme of the pneumatic valve [41].

The position of the piston is controlledby regulating the pressure of the pneumatic ports to fill or evacuate the top and bottom chambers. The degradation mechanism of the valve is considered as the external leak at the actuator connections to the bottom pneumatic port due to corrosion, and is modeled by a PBM. It is much more significant than the other degradation mechanisms according to the results shown in [41]. The valve is considered failed when the size of the external leak exceeds a predefined $D_{b}^{*}$. The PBM is used by EDF experts for degradation modeling, due to limited statistical degradation data on the valve behavior.

\subsection{PDMP for the system}

The degradation of the valve $\boldsymbol{L}=\left\{L_{1}\right\}$ is described by PBM and the degradation of the pump $\boldsymbol{K}=\left\{K_{1}\right\}$ is described by MSM. The degradation processes of the whole system are modeled by PDMP as follows:

$$
\vec{Z}(t)=\left(\begin{array}{c}
D_{b}(t) \\
Y_{p}(t)
\end{array}\right) \in \mathbb{R}^{+} \times S_{p}
$$

where $Y_{p}(t)$ denotes the degradation state of the pump at time $t$ and $D_{b}(t)$ denotes the area of the leak hole at the bottom pneumatic port of the valve at time $t$. The space of the failure states 
of $\vec{Z}(t)$ is $\mathcal{F}=[0,+\infty) \times\left\{0^{\prime}\right\} \cup\left[D_{b}^{*},+\infty\right) \times\{1,2,3\}$. The development of the leak size is described by:

$$
\dot{D_{b}}(t)=\omega_{b}\left(1+\beta_{Y_{p}(t)}\right)
$$

where $\omega_{b}$ is the original wear coefficient and where $\beta_{Y_{p}(t)}$ is the relative increment of the developing rate of the external leak caused by the vibration of the pump at the degradation state $Y_{p}=2$ or 1 . The parameter values related tothe systemdegradation processes under accelerated aging conditionsand to the maintenance tasks are presented in Table I. For confidentiality reasons, the values presented below are fictitious.

Table I Parameter values related to PDMP and the maintenance tasks

\begin{tabular}{|c|c|}
\hline Parameter & Value \\
\hline$\omega_{b}$ & $1 \mathrm{e}-8 \mathrm{~m}^{2} / \mathrm{s}$ \\
\hline$\beta_{2}$ & $10 \%$ \\
\hline$\beta_{1}$ & $20 \%$ \\
\hline$\lambda_{32}$ & $3 \mathrm{e}-3 \mathrm{~s}-1$ \\
\hline$\lambda_{21}$ & $3 \mathrm{e}-3 \mathrm{~s}-1$ \\
\hline$\lambda_{10}$ & $3 \mathrm{e}-3 \mathrm{~s}-1$ \\
\hline$D_{b}^{*}$ & $1.06 \mathrm{e}-5 \mathrm{~m}^{2}$ \\
\hline$T_{L_{1}}$ & $1000 \mathrm{~s}$ \\
\hline$T_{K_{1}}$ & $1000 \mathrm{~s}$ \\
\hline$H_{L_{1}}$ & {$\left[8 \mathrm{e}-6, D_{b}^{*}\right) \mathrm{m}^{2}$} \\
\hline$H_{K_{1}}$ & $\{1,2\}$ \\
\hline
\end{tabular}

The system reliability at timet can be calculated as follows:

$$
R(t)=P\left[\left(D_{b}(s)<D_{b}^{*}\right) \cap\left(Y_{p}(s) \neq 0\right), \forall s \leq t\right]
$$

The component IMs for the valve and the pump are given in eq. (29) and eq. (30), respectively, as follows:

$$
\left.C I_{V}(t)=\int_{\mathbb{R}^{+}} f_{\overrightarrow{D_{V}}(t)}(x) \mid P\left[\left(D_{b}(s)<D_{b}^{*}\right) \cap\left(Y_{p}(s) \neq 0\right), \forall s \leq t \mid D_{b}(t)=x\right)\right]-
$$




$$
\left.C I_{P}(t)=\sum_{i=0}^{3} P\left[Y_{p}(t)=i\right] \mid P\left[\left(D_{b}(s)<D_{b}^{*}\right) \cap\left(Y_{p}(s) \neq 0\right), \forall s \leq t \mid Y_{p}(t)=i\right)\right]-R(t) \mid
$$

Then, by using the proposed numerical method introduced in section 4 , the values of the above equations can be calculated.

\subsection{Results}

The reliabilitiesof the whole system and the two components over a time horizon of $T_{\text {miss }}=2000$ s, regarded as the mission time under accelerated conditions, are shown in Fig. 5. We can see from the figure that before around 870s (point A), thesystem reliabilityis basicallydetermined by the pump reliability, since the valve is highly reliable. After that, the sharp decrease of the reliability of the valvedue to degradation drives that of the system reliability,until the execution of the inspection tasks for the two components at 1000s. Because of the preventive maintenance, the failures of the system, the valve and the pump are mitigated.

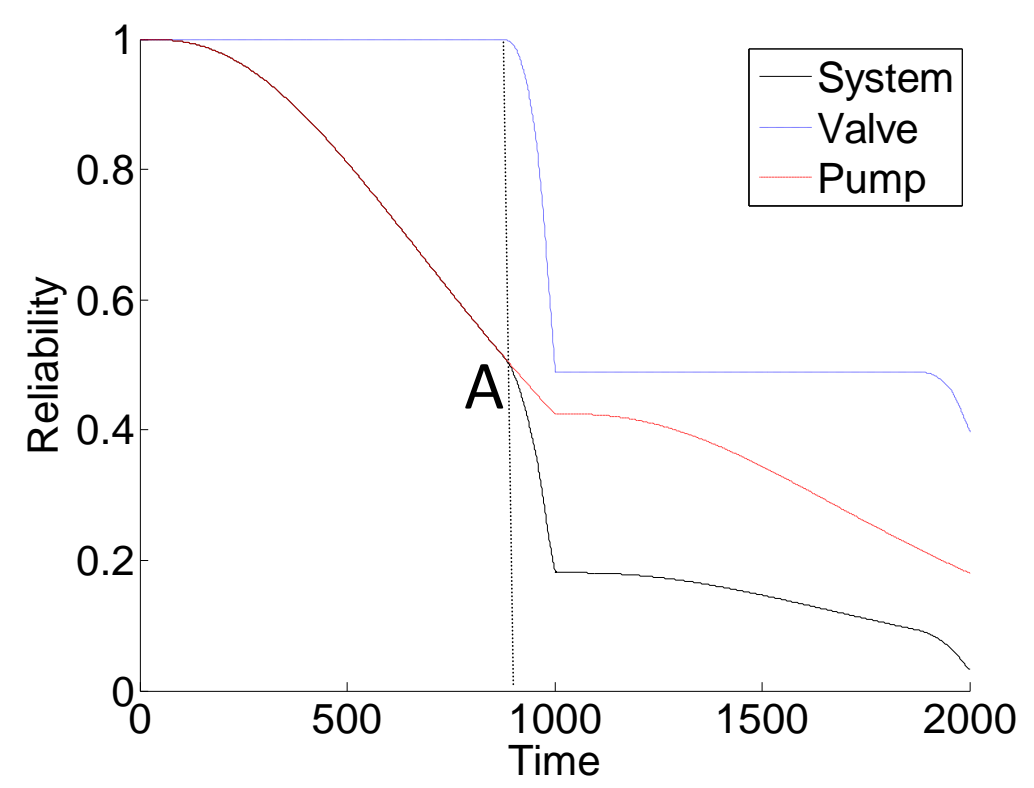

Fig. 5. The reliabilities of the system, the valve and the pump

The components IMs are shown in Fig. 6.Before around 400s (point B), the IMs of the two components are relatively close.Although the system reliability is dominated by the reliability of the pump, theprobability of the pump at state 0over the time horizon is limited to 
a very small value due to the corrective maintenance shown in Fig. 7, which canlimitthe component IM. After around 870s (point C), the pump IM experiences a sharp decrease while that of the valve experiences a sharp increase until 1000s, due to the evolution shown in Fig. 5.After the preventive maintenance is implemented, the difference between the components IMs begins to reduce. Then, one can conclude that attentionshould be focused onthe pump before 1000s and on the valve afterwards, to achievehigher levels of system reliability.

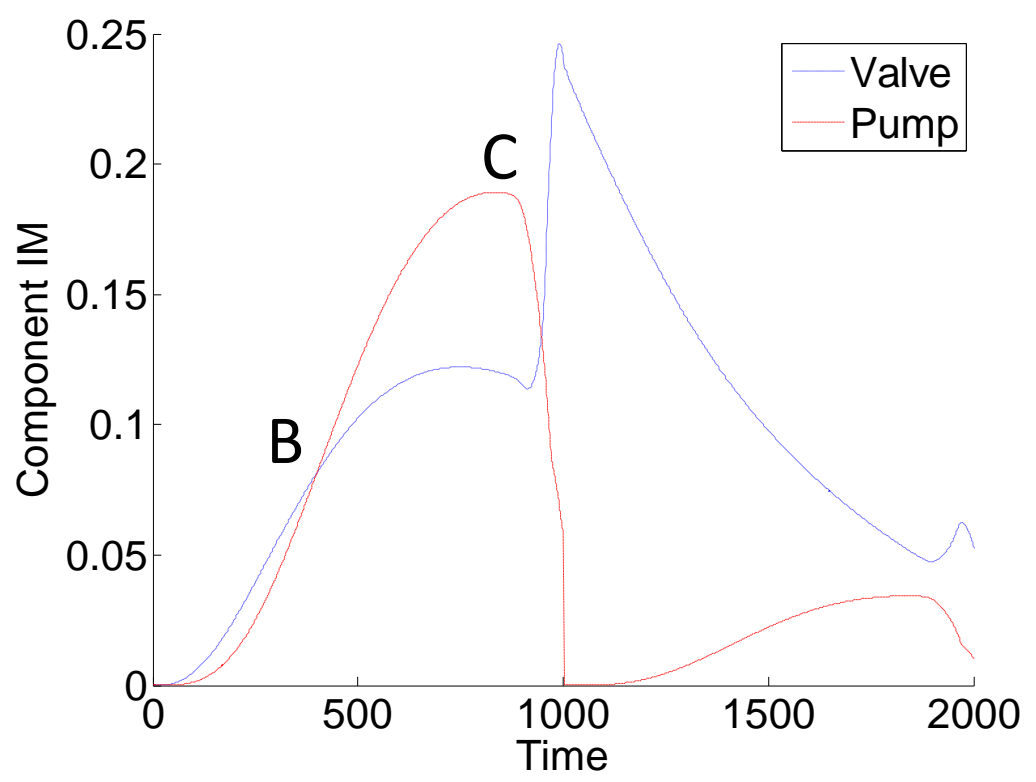

Fig. 6. The valve and pump IMs 


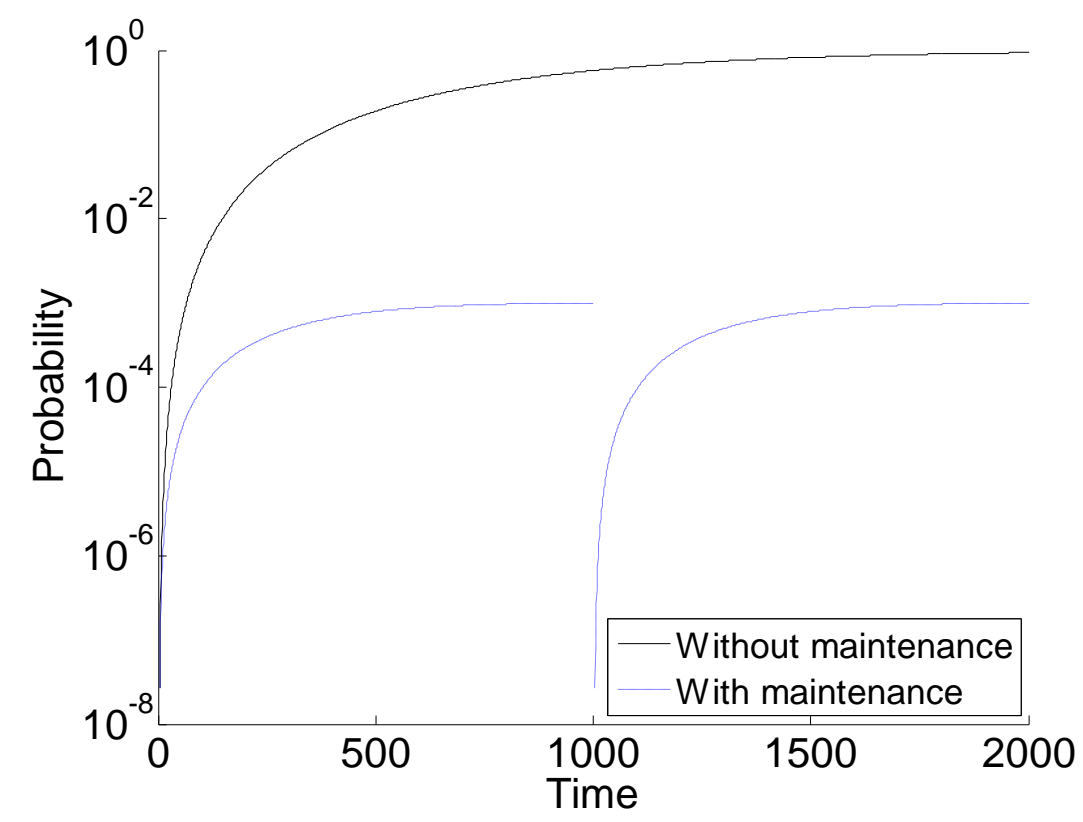

Fig. 7. The probability of the pump at state 0 (failure)

The reliabilities of the whole system and the two components over a time horizon of $T_{\text {miss }}=2000 \mathrm{~s}$ without maintenance are shown in Fig. 8. Before 1000s, the situationsare the same as with maintenance (Fig. 5). The sharp decrease of the reliability of the valve, thencontinues due to the lack of preventive maintenance, andthe valve reaches failure after around $1060 \mathrm{~s}$, and the system fails too.

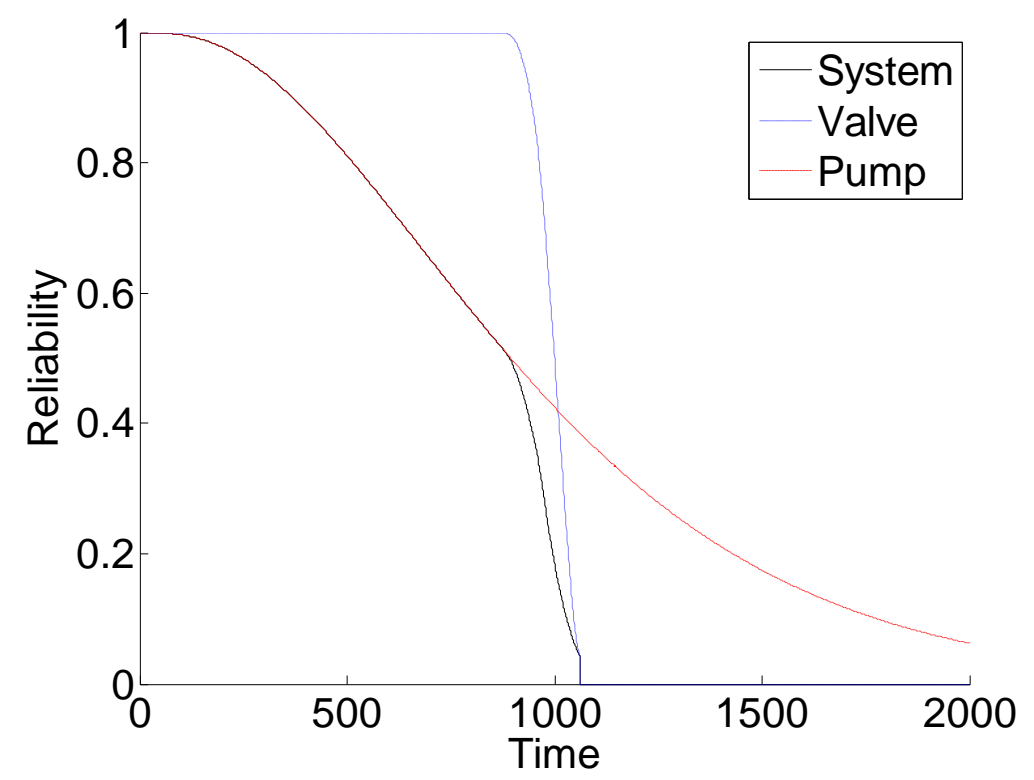


Fig. 8. The reliabilities of the system, valve and pump without maintenance

The related component IMs are shown in Fig. 9. From the figure, we can see thatthe criticality of the pump is higher than that of the valve most of the time until around 1015s (point E). Due to the absence of preventive maintenance, the system reliability quickly decreases to zero afterwards, which leads the components IMs toquickly decrease to zero. The gap between the two curves isdue to the difference between the reliabilities of the two components, andreaches its maximum value at around $875 \mathrm{~s}$ (point D), when the valve starts to contribute to the system failure.

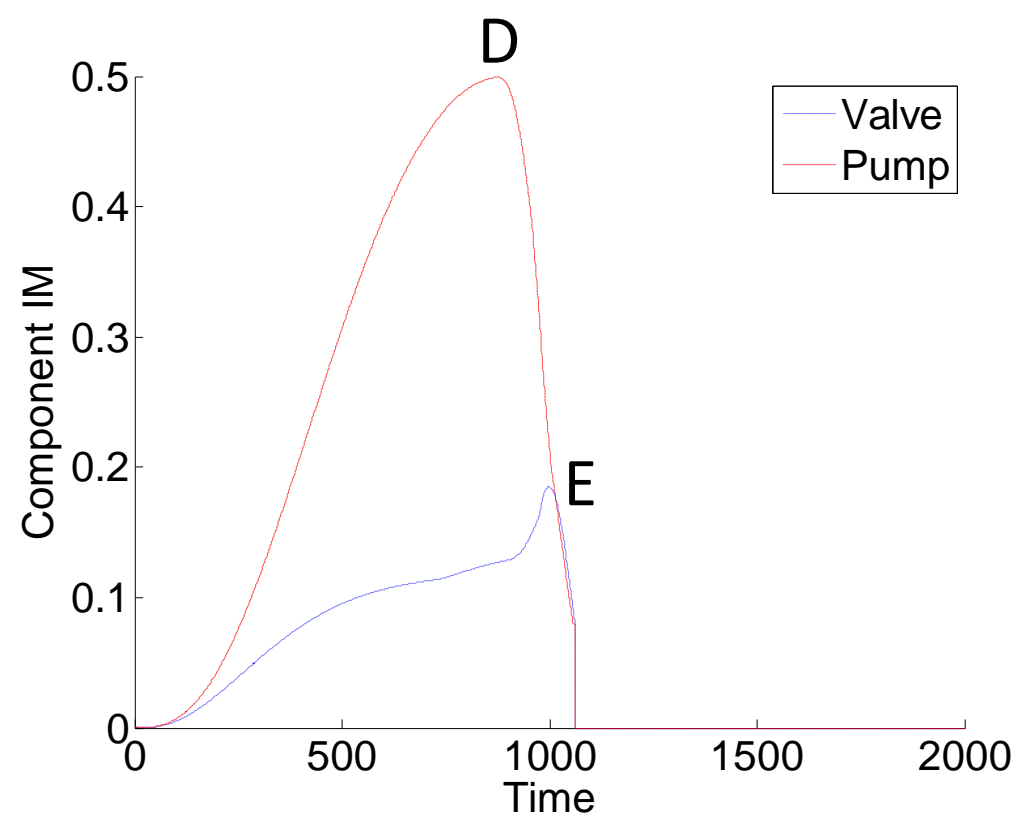

Fig. 9. The valve and pump IMs without maintenance

Finally, the reliabilities of the whole system and the two components over a time horizon of $T_{\text {miss }}=2000$ s, without degradation dependency,are shown in Fig. 10.The system reliability is determined by the reliability of the pump since the valve is highly reliable. The IMs of the two components are shown in Fig. 11. The IM of the pump experiences a sudden change due to the preventive maintenance at 1000s, while that of the valve is always equal to zero. 


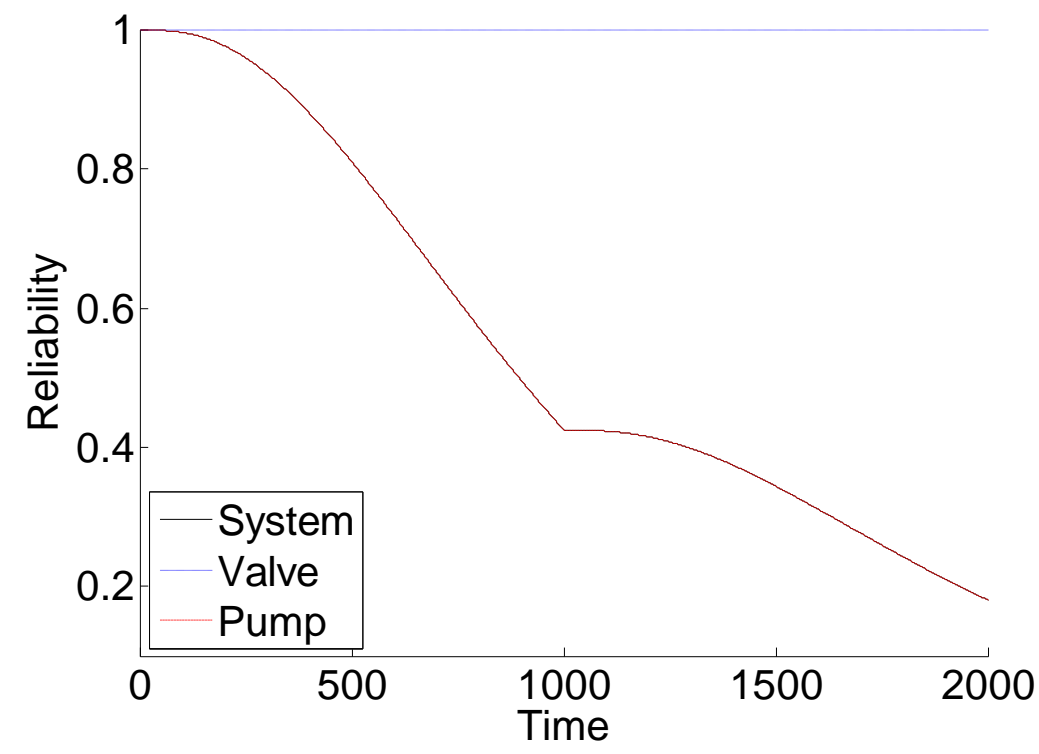

Fig. 10. The reliabilities of the system, the valve and the pump without degradation dependency

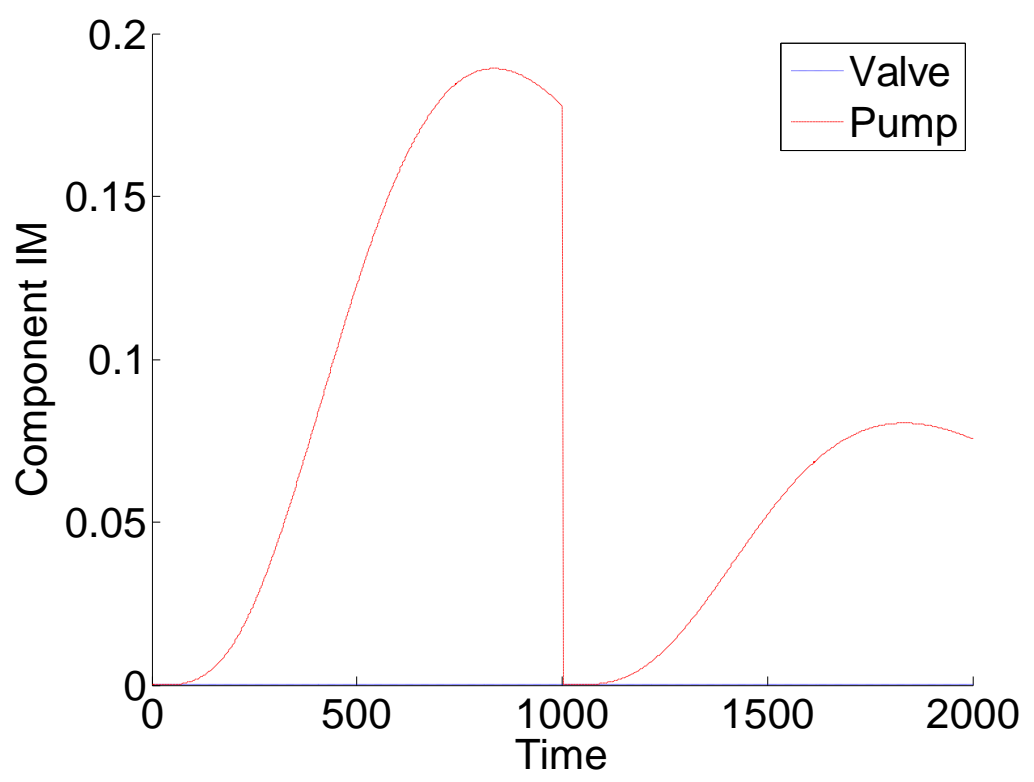

Fig. 11. The valve and pump IMs without degradation dependency

To investigate the impacts of the periods of the inspection tasks, the IMs of the two components with different inspection periods are shown in Fig. 12. We have tested two 
settings $T_{L_{1}}=T_{K_{1}}=500 \mathrm{~s}$ and $T_{L_{1}}=T_{K_{1}}=250 \mathrm{~s}$. From the figure, we can see that the IM of the valve is always equal to zero since it is highly reliable andthat the increase of the inspection frequency can reduce the IM of the pump.

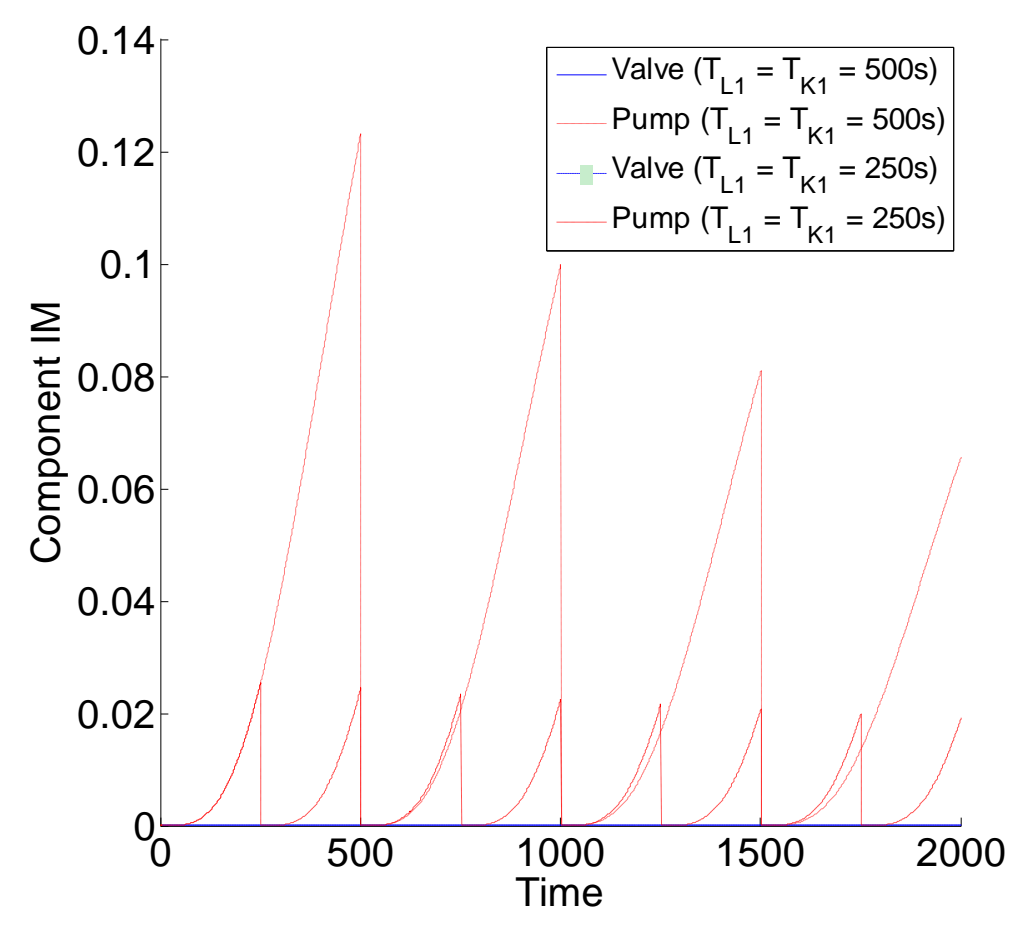

Fig. 12. The valve and pump IMs with different inspection periods

\section{CONCLUSION}

In this paper, we consider components with multiple competing degradation processes modeled by PBMs and MSMs. The PDMP modeling framework is employed to incorporate multiple dependent competing degradation processes and maintenance policies. To quantify the importance of different components within a system, MAD IM has been extended to accommodate components whose (degradation) states are determined by both discrete and continuous processes. The extended IM can provide timely feedbacks on the criticality of a component with respect to the system reliability. The degradation dependencies within one component and among different components, and two types of maintenance tasks (conditionbased preventive maintenance by periodic inspections and corrective maintenance) have been 
taken into account. A quantification method based on the FV approach has been developed and illustrated in the application to a case study of a portion of an emergency system (the RHRS) from real-world nuclear power plants. The illustrative example shows that the extended IM can effectively estimate the criticality of different components under the conditions of interest.

As future work, it would be interesting to study how the sensitivity indices of the parameters of a component relate to the importance indices of that component, within a GSA framework.

\section{REFERENCES}

[1] W. Kuo and X. Zhu, "Some recent advances on importance measures in reliability," Reliability, IEEE Transactions on, vol. 61, pp. 344-360, 2012.

[2] H. Peng, D. W. Coit, and Q. Feng, "Component reliability criticality or importance measures for systems with degrading components," Reliability, IEEE Transactions on, vol. 61, pp. 4-12, 2012.

[3] Y. Wang and H. Pham, "Modeling the dependent competing risks with multiple degradation processes and random shock using time-varying copulas," Reliability, IEEE Transactions on, vol. 61, pp. 13-22, 2012.

[4] J. Lei, F. Qianmei, and D. W. Coit, "Reliability and Maintenance Modeling for Dependent Competing Failure Processes With Shifting Failure Thresholds," Reliability, IEEE Transactions on, vol. 61, pp. 932-948, 2012.

[5] N. Rasmekomen and A. K. Parlikad, "Maintenance Optimization for Asset Systems With Dependent Performance Degradation," Reliability, IEEE Transactions on, vol. 62, pp. 362-367, 2013.

[6] S. Albin and S. Chao, "Preventive replacement in systems with dependent components," Reliability, IEEE Transactions on, vol. 41, pp. 230-238, 1992.

[7] L. Jiang, Q. Feng, and D. W. Coit, "Reliability and maintenance modeling for dependent competing failure processes with shifting failure thresholds," Reliability, IEEE Transactions on, vol. 61, pp. 932-948, 2012.

[8] E. Keedy and Q. Feng, "A physics-of-failure based reliability and maintenance modeling framework for stent deployment and operation," Reliability Engineering \& System Safety, vol. 103, pp. 94-101, 2012.

[9] A. Lisnianski and G. Levitin, Multi-state system reliability: assessment, optimization and applications: World scientific, 2003.

[10] M. Chookah, M. Nuhi, and M. Modarres, "A probabilistic physics-of-failure model for prognostic health management of structures subject to pitting and corrosion-fatigue," Reliability Engineering \& System Safety, vol. 96, pp. 1601-1610, 2011.

[11] S. D. Unwin, P. P. Lowry, R. F. Layton, P. G. Heasler, and M. B. Toloczko, "Multi-state physics models of aging passive components in probabilistic risk assessment," in International Topical Meeting on Probabilistic Safety Assessment and Analysis (PSA 2011), Wilmington, North Carolina, 2011, pp. 161-172. 
[12] Y.-H. Lin, Y.-F. Li, and E. Zio, "Fuzzy Reliability Assessment of Systems with Multiple Dependent Competing Degradation Processes," Fuzzy Systems, IEEE Transactions on (In press).

[13] C. M. Rocco and E. Zio, "Global sensitivity analysis in a Multi-state Physics Model of Component Degradation based on a hybrid State-Space Enrichment and Polynomial Chaos Expansion approach," Reliability, IEEE Transactions on, vol. 62, pp. 781-788.

[14] Z. W. Birnbaum, "On the importance of different components in a multicomponent system," Multivariable analysis 2, New York: Academic Press, pp. 581-592, 1969.

[15] W. Kuo and X. Zhu, "Relations and generalizations of importance measures in reliability," Reliability, IEEE Transactions on, vol. 61, pp. 659-674, 2012.

[16] R. E. Barlow and F. Proschan, "Importance of system components and fault tree events," Stochastic Processes and their Applications, vol. 3, pp. 153-173, 1975.

[17] D. Vasseur and M. Llory, "International survey on PSA figures of merit," Reliability Engineering \& System Safety, vol. 66, pp. 261-274, 1999.

[18] W. Kuo and X. Zhu, Importance measures in reliability, risk, and optimization: principles and applications: John Wiley \& Sons, 2012.

[19] M. J. Armstrong, "Reliability-importance and dual failure-mode components," Reliability, IEEE Transactions on, vol. 46, pp. 212-221, 1997.

[20] W. S. Griffith, "Multistate reliability models," Journal of Applied Probability, pp. 735-744, 1980.

[21] S. Wu and L.-Y. Chan, "Performance utility-analysis of multi-state systems," Reliability, IEEE Transactions on, vol. 52, pp. 14-21, 2003.

[22] S. Si, H. Dui, X. Zhao, S. Zhang, and S. Sun, "Integrated importance measure of component states based on loss of system performance," Reliability, IEEE Transactions on, vol. 61, pp. 192-202, 2012.

[23] S. Si, G. Levitin, H. Dui, and S. Sun, "Component state-based integrated importance measure for multi-state systems," Reliability Engineering \& System Safety, vol. 116, pp. 75-83, 2013.

[24] H. Dui, S. Si, L. Cui, Z. Cai, and S. Sun, "Component Importance for Multi-State System Lifetimes With Renewal Functions," Reliability, IEEE Transactions on, vol. 63, pp. 105-117, 2014.

[25] E. Zio and L. Podofillini, "Monte Carlo simulation analysis of the effects of different system performance levels on the importance of multi-state components," Reliability Engineering \& System Safety, vol. 82, pp. 63-73, 2003.

[26] G. Levitin, L. Podofillini, and E. Zio, "Generalised importance measures for multi-state elements based on performance level restrictions," Reliability Engineering \& System Safety, vol. 82, pp. 287-298, 2003.

[27] J. E. Ramirez-Marquez and D. W. Coit, "Composite importance measures for multi-state systems with multi-state components," Reliability, IEEE Transactions on, vol. 54, pp. 517-529, 2005.

[28] N. Gebraeel, "Prognostics-Based Identification of the Top-Units in a Fleet," Automation Science and Engineering, IEEE Transactions on, vol. 7, pp. 37-48, 2010.

[29] X. Liu, K. N. Al-Khalifa, E. A. Elsayed, D. W. Coit, and A. S. Hamouda, "Criticality measures for components with multi-dimensional degradation," IIE Transactions, 2013.

[30] S. Song, D. W. Coit, Q. Feng, and H. Peng, "Reliability Analysis for Multi-Component Systems Subject to Multiple Dependent Competing Failure Processes," 2014.

[31] S. Iyer, "The Barlow-Proschan importance and its generalizations with dependent components," Stochastic processes and their applications, vol. 42, pp. 353-359, 1992.

[32] B. Natvig, "Measures of component importance in nonrepairable and repairable multistate strongly coherent systems," Methodology and Computing in Applied Probability, vol. 13, pp. 523-547, 2011. 
[33] C. Cocozza-Thivent, "Processus de renouvellement markovien, Processus de Markov déterministes par morceaux," Online book available on the webpage: http://perso-math. univ-m/v. fr/users/cocozza. christiane/recherche-pageperso/PresentationRMetPDMP. html, 2011.

[34] C. Cocozza-Thivent, R. Eymard, and S. Mercier, "A finite-volume scheme for dynamic reliability models," IMA journal of numerical analysis, vol. 26, pp. 446-471, 2006.

[35] R. Coudray and J. Mattei, "System reliability: An example of nuclear reactor system analysis," Reliability Engineering, vol. 7, pp. 89-121, 1984.

[36] M. Marseguerra and E. Zio, "Monte Carlo approach to PSA for dynamic process systems," Reliability Engineering \& System Safety, vol. 52, pp. 227-241, 1996.

[37] R. Eymard and S. Mercier, "Comparison of numerical methods for the assessment of production availability of a hybrid system," Reliability Engineering \& System Safety, vol. 93, pp. 168-177, 2008.

[38] M. H. Davis, Markov models and optimization vol. 49: CRC Press, 1993.

[39] S. Zhang, M. Hodkiewicz, L. Ma, and J. Mathew, "Machinery condition prognosis using multivariate analysis," in Engineering Asset Management: Springer, 2006, pp. 847-854.

[40] P. Moussou, S. Cambier, D. Lachene, S. Longarini, L. Paulhiac, and V. Villouvier, "Vibration investigation of a French PWR power plant piping system caused by cavitating butterfly valves," ASME-PUBLICATIONS-PVP, vol. 420, pp. 99-106, 2001.

[41] M. Daigle and K. Goebel, "A model-based prognostics approach applied to pneumatic valves," International journal of prognostics and health management, vol. 2, p. 008, 2011.

Yan-Hui Linhas been a doctoral student at Chair System Science and the Energy Challenge, FondationElectricité de France (EDF), CentraleSupélec, Université Paris-Saclay, France since August 2012. He received the B.Sc. degree in Applied Mathematics from Beihang University, China, the M.Sc. degree in Applied Mathematics from Ecole Centrale Paris, France and the M.Sc. degree in Aircraft Design from Beihang University, China, in 2010, 2012 and 2013, respectively. His research interests are in reliability, degradation and maintenance modeling, Monte Carlo simulation, and optimization under uncertainty.

Yan-Fu Li is an Assistant Professor at Chair System Science and the Energy Challenge, FondationElectricité de France (EDF), CentraleSupélec, Université Paris-Saclay, France. Dr. Li completed his $\mathrm{PhD}$ research in 2009 at National University of Singapore, and went to the University of Tennessee as a research associate. His current research interests include reliability modeling, uncertainty analysis, evolutionary computing, and Monte Carlo simulation. He is the author of more than 30 publications, all in refereed international journals, conferences, and books. 
Enrico Zioreceived the Ph.D. degree in nuclear engineering from Politecnico di Milano and MIT in 1995 and 1998, respectively. He is currently Director of the Chair System Science and the Energy Challenge, FondationElectricité de France (EDF), CentraleSupélec, Université Paris-Saclay, France, and full professor at Politecnico di Milano, Italy. His research focuses on the characterization and modeling of the failure/repair/maintenance behavior of components, complex systems and their reliability, maintainability, prognostics, safety, vulnerability and security, Monte Carlo simulation methods, soft computing techniques, and optimization heuristics. 